\title{
Lobular capillary hemangioma in the hard palate: A rare case
}

\author{
Gaurav Khutwad ${ }^{1}$, Heena Sayed ${ }^{2, *}$, Harshad Bhagwat ${ }^{3}$, Aatif Sayed ${ }^{4}$ \\ ${ }^{1,4}$ Senior Lecturer, ${ }^{2}$ Chief Resident, ${ }^{3}$ Reader, Dept. of Oral and Maxillofacial Surgery, M. A. Rangoonwala College of Dental \\ Science and Research Centre, Pune, India
}

*Corresponding Author:

Email: Hee.sayed@hotmail.com

\begin{abstract}
Haemangioma of the oral cavity is an uncommon pathological entity, but the head and neck are the common sites. These lesions occur in infants and children. They exhibit a rapid endothelial cell proliferative phase followed by involution over time. The tumour presents as a flat or raised reddish lesion, usually solitary. Lobular capillary haemangioma arises from the capillary tissue and commonly occurs in the skin. Its occurrence in the hard palate is rare and hence, the lesion should be distinguished from other benign lesions of the oral cavity. The purpose of this article is to report an unusual case of a benign tumour in a young girl occurring on the hard palate which was clinically diagnosed as traumatic fibroma and was treated by surgical excision. The excised mass was sent for histopathological examination and a diagnosis of lobular capillary haemangioma was established.
\end{abstract}

Keywords: Lobular capillary haemangioma, hard palate, benign lesion, histopathology.

\section{Introduction}

Oral Haemangioma is a benign tumor of the blood vessels. In 1982, a classification of vasoformative tumors was given by Mulliken and Glowacki, which was divided into two broad groups: Haemangioma and vascular malformations. ${ }^{1}$ However, haemangioma is not present at birth whereas vascular malformation is present at birth. Vascular malformation results from the anomalous development of vascular plexus. Although haemangioma is an uncommon tumor of the oral cavity, they usually occur in lips, cheeks, and tongue. Haemangioma is broadly classified into capillary, venous, arteriovenous and lymphatic haemangioma. These lesions are more common in whites, but it rarely occurs in dark-skinned individuals. It is found to be about three times more common in females than in males and frequently involves the early age group. ${ }^{2}$ Their differential diagnosis is based on the clinical appearance. Depending on the site of occurrence, it may interfere with the function such as mastication in case of oral mucosa involvement, breathing and airway obstruction in case of nasal or laryngeal mucosa involvement. There has been no evidence of loss of sensation in the involved area.

Lobular capillary haemangioma was first described as human botryomycosis by Poncet and Dor in $1897 .{ }^{3}$ Lobular capillary haemangioma is a rapidly growing vascular benign lesion of unknown etiology, and it usually occurs in the skin and mucous membranes as a pedunculated red or bluish nodule, which is prone to ulceration or bleeding. Histological features will show superficial infiltration by a large number of acute and chronic inflammatory cells, but this is seen only in ulcerated lesions. Focal cytological atypia may be seen as a result of degeneration. It is often clinically confused with other benign lesions of the oral cavity and needs to be differentiated from them. Therefore, its histopathological assessment will determine an accurate and more satisfactory diagnosis. The classification of haemangioma is based on histological appearance and classified as capillary, mixed cavernous, or sclerosing variety that tends to undergo fibrosis. ${ }^{4}$

\section{Case Report}

A 10 years old Indian girl reported to our institute with a chief complaint of swelling in the anterior maxilla since 2 months (Fig. 1). The patient was asymptomatic before 2 months, since then she experienced a swelling in that region, due to mild trauma. The traumatic factor was an attempt to remove the food particle lodged in that area. The swelling increased in size due to continuous repeated trauma during mastication (Fig. 2). The growth was asymptomatic. It then started decreasing in size after the $10^{\text {th }}$ day of its appearance (on taking medication from a local dentist) to achieve the size with which the patient reported to us. The swelling was non-tender but patient experienced pain while biting on hard food. There was no history of discharge, or draining sinus, however, there was bleeding from the swelling 4 days before she reported. The patient had no associated fever or nasal discharge. The patient was a mouth breather and revealed no history of nail biting or thumb-sucking.

On general examination, the patient was well oriented and had a positive behaviour rating. Patient's vital signs were within normal limits and no evidence of any underlying disease. Local intraoral examination revealed a $3 * 1 \mathrm{~cm}$ swelling on the palatal aspect extending from 12 to 22 region. The colour of the overlying mucosa reddish pink colour and had a textured surface. On palpation, a non-tender, nonfluctuant soft tissue swelling was felt which was firm in consistency with a pedunculated base. There was no discharge, bleeding or draining sinus associated with the swelling. Based on the clinical examination, a provisional diagnosis of traumatic oral fibroma was 
made with a differential diagnosis of giant cell fibroma, peripheral giant cell granuloma or pyogenic granuloma.

An IOPA (Intra-Oral Peri-Apical) radiograph was advised which showed no significant finding and no bone loss (Fig. 4). No further radiograph was advised to reduce exposure to the developing tooth buds. An excisional biopsy was advised. A routine blood investigation was carried out, which showed no evidence of infection. The soft tissue overgrowth was excised completely from the base under local anaesthesia (Fig. 3) with appropriate bleeding control measures i.e. pressure and electrocautery, and was sent for histopathological examination. Macroscopic examination showed a single nodular lesion, brownish in colour and firm in consistency (Fig. 5).

Histopathological examination (Fig. 6) showed a nodular proliferation rich in proliferating capillary sized blood vessels mixed with inflammatory cells mainly plasma cells. There was no cellular atypical seen lining the blood vessels. Overlying mucosa showed ulceration. There was no evidence of malignancy. These features led to a diagnosis of Lobular Capillary Haemangioma.

Careful inspection of the patient and the excised site was carried out thereafter. There was no reactionary or secondary haemorrhage reported from the patient's end. The patient was recalled for frequent follow-ups up to a year to look for recurrence or any malignancy. The wound healing was satisfactory (Fig. 7) and no recurrence was noted.

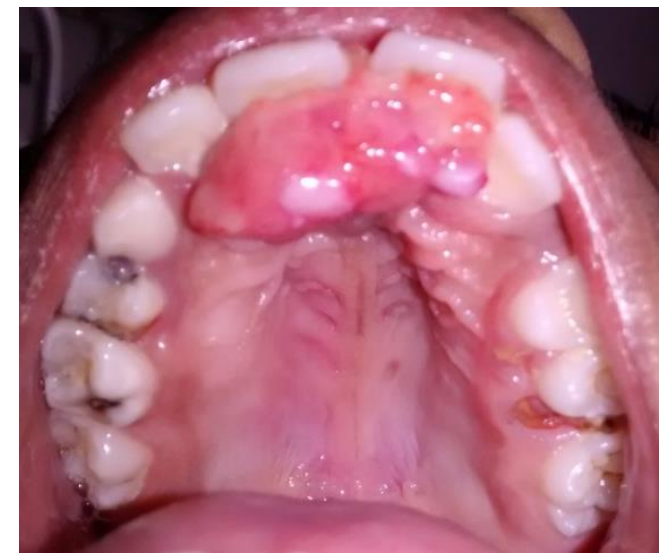

Fig. 1: A reddish-pink, firm swelling seen on the anterior aspect of the hard palate

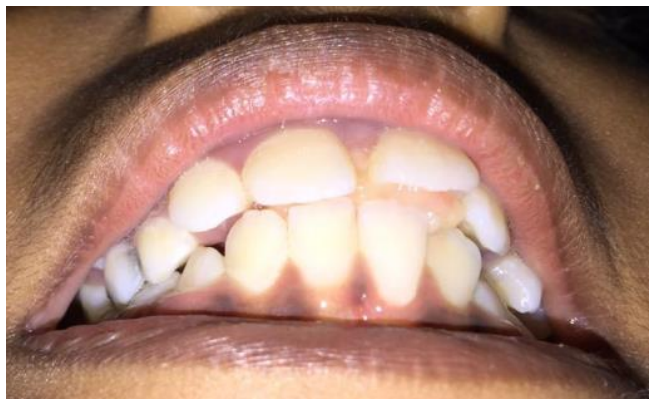

Fig. 2: Repeated trauma from mastication

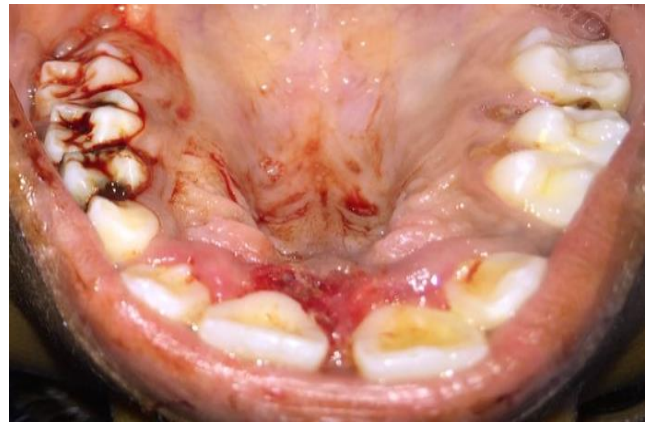

Fig. 3: Excision of the soft tissue mass from the base with cauterization

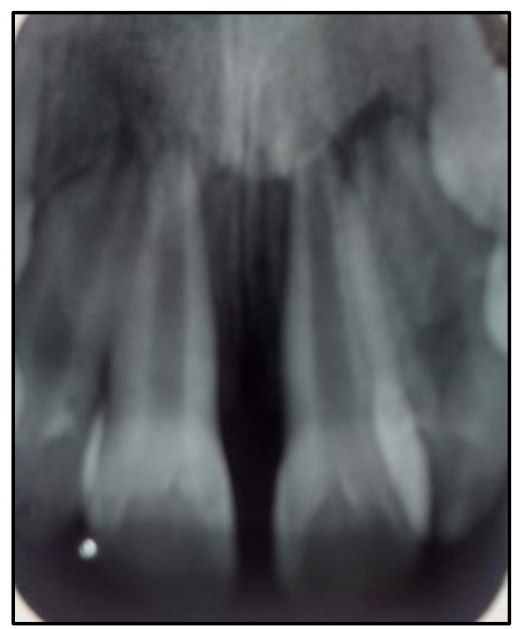

Fig. 4: IOPA of the area showing no bone loss

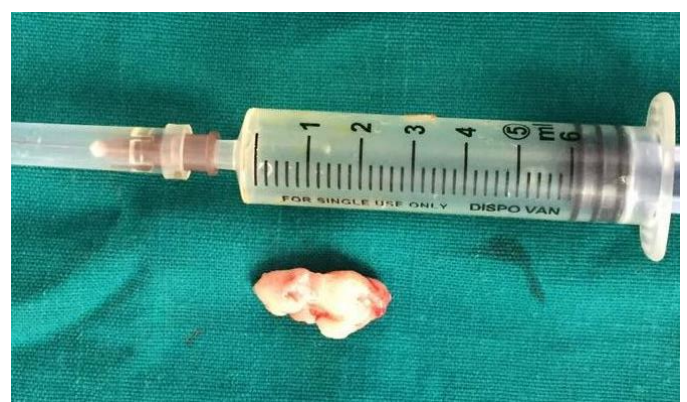

Fig. 5: Excised specimen

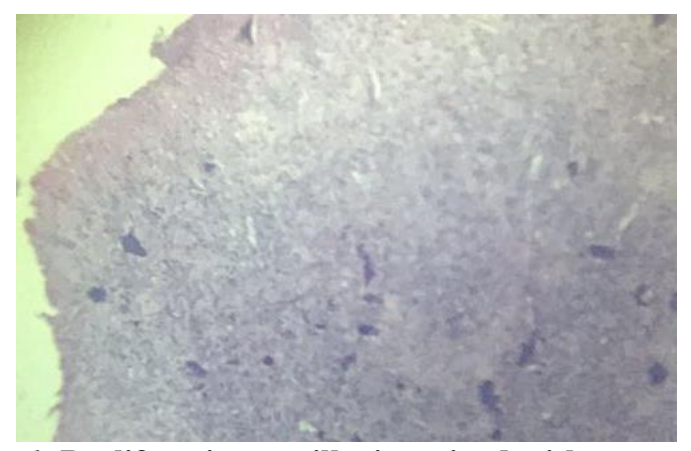

Fig. 6: Proliferating capillaries mixed with inflammatory cells 


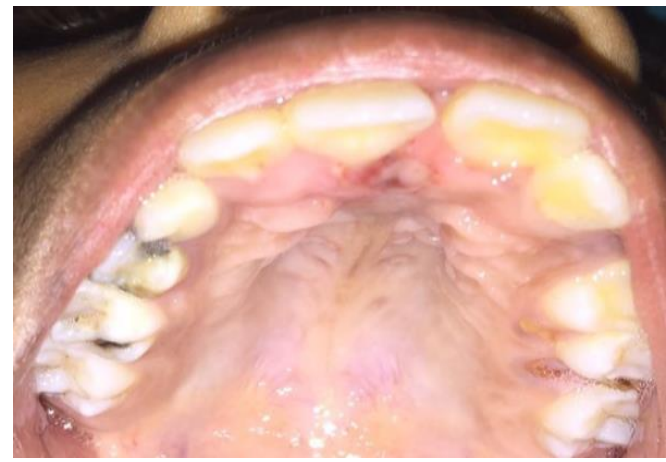

Fig. 7: Post-operative healing on the $3^{\text {rd }}$ day

\section{Discussion}

The most common location of haemangioma is the head and neck, which accounts for $60 \%$ of all cases. $80 \%$ of haemangioma occur as a solitary lesion, and remaining $20 \%$ as multiple tumors. Lobular capillary haemangioma, a histological variant of haemangioma is also known as pyogenic granuloma or botryomycoma. Pyogenic granuloma is a misnomer, as this condition neither clinically presents with pus formation, nor does it represent a granuloma histologically. Based on the histopathological examination, it is termed as lobular capillary hemangioma. ${ }^{5}$ Although, it is difficult to differentiate between a true haemangioma of infancy and a pyogenic granuloma in a light microscope, the latter, exhibits immunocytochemical and ultrastructural differences. ${ }^{6}$

Clinically, it is quite challenging to differentiate this pathology from other benign tumours of the oral cavity. A provisional diagnosis of traumatic oral fibroma was given owing to the traumatic etiological factor and that fibroma is a reactive hyperplasia of fibrous connective tissue ${ }^{7}$ in response to trauma which in this case could be an attempt to remove the stuck food particle, or local irritation which could be the deep overbite. Fibroma is a true neoplasm of the connective tissue which occurs as a part of the inflammatory reaction due to chronic oral irritation. There is an increase in the number of cells due to increased mitotic activity and the lesion does not regress on the removal of irritants. $^{2}$ A differential diagnosis of giant cell fibroma could be considered due to the similar clinical presentation, i. e. the young age and a female predilection. However, it is usually small (less than 1 $\mathrm{cm}$ in diameter) and is not associated with chronic irritation. Peripheral giant cell granuloma has a rapid growth rate in contrast to the above-mentioned pathology which increased gradually in size. Pyogenic granuloma is another pathology which usually involves a history of trauma and commonly occurs in the children and young adults with a female predilection. ${ }^{7}$

Lobular capillary haemangioma is a benign, richly vascularized inflammatory lesion of the skin or mucosa. ${ }^{8}$ A colour change can be observed on pressure which returns to original colour on the removal of pressure. A clinic-pathological study done by Patrice et al in a series of 178 patients, reported a $21 \%$ rate of mucosal involvement of the oral cavity and conjunctivae. $^{5}$ Very few cases of capillary haemangioma on the hard palate have been reported in the literature so far.,9-14 Ozcan et al reported a case of intra-nasal lobular capillary hemangioma in a 6-yearold girl who presented with epistaxis and nasal obstruction. This entity to occur in the nasal cavity is a rare and can be managed by total excision endoscopically ${ }^{15}$. A case similar to ours was reported by Satish et al wherein capillary hemangioma was diagnosed in the maxillary anterior region of a 10-year old boy after histopathological evaluation. ${ }^{16}$

In the undifferentiated capillary network stage, there is an arrest in the development of mesenchyme primordia. As differentiation progresses, the primitive vessels penetrate deep into the subcutaneous layer, the muscle, or the bone tissue giving rise to capillary hemangiomas. $^{2}$ When the epithelial basement membrane has been penetrated by these vessels, bleeding occurs during its excision. It can mimic a malignant pathology. Its rich vascularization requires caution during biopsy, with appropriate bleeding control measures like pressure and suturing. The risk of recurrence requires excision to be complete and curettage of the underlying tissue is recommended. ${ }^{8}$ As the lesion was small, non-tender with no bone involvement as was evident in the radiograph and did not have any life-threatening possibility, it could be carried out by simple excision under local anaesthesia with appropriate bleeding control measures.

Other treatment options include ligation and excision, artificial ulceration, electrocautery and thermocautery, sclerosant therapy, radiation and compression depending upon the clinical features and the anatomical considerations. In recent days, management consists of 'primum non nocere' i.e. spontaneous involution, steroid therapy and chemotherapy. ${ }^{6}$ Complications occur in about $20 \%$ of haemangioma, the most common complication being ulceration, which may or may not be accompanied with secondary infection. Another common complication, haemorrhage may be noted, though significant blood loss does not usually occur. ${ }^{4}$

Capillary haemangioma occurring on the palate is a rare site, and so it can be confused easily with other lesions. Hence, a conjugation of complete clinical and histopathological evaluation is necessary as a histopathological evaluation will give a more accurate form of diagnosis. In order to treat such lesions, dental practitioners and oral surgeons should be aware of the risks they can come across during its surgical procedure, bleeding being one of them and should be prepared to manage the same.

No Funding: This report did not receive any specific grant from funding agencies in the public, commercial or not-for-profit sector. 


\section{References}

1. Mulliken JB, Glowacki J. Hemangiomas and vascular malformations in infants and children: a classification based on endothelial characteristics. Plast Reconstr Surg 1982;69:412-20.

2. Shafer WG, Hine MK, Levy BM. A Textbook of Oral Pathology. 4th ed. Philadelphia: WB Saunders; 1983;359-60.

3. Poncet A, Dor L. Botyromycose Humaine Rev Chir (Paris) 1987;18:996.

4. Açikgöz A, Sakallioglu U, Ozdamar S, Uysal A. Rare benign tumours of the oral cavity- capillary hemangioma of palatal mucosa: a case report. Int J Paediatr Dent 2000;10(2):161-5.

5. Patrice SJ, Wiss K, Mulliken JB. Pyogenic granuloma (lobular capillary hemangioma): a clinicopathologic study of 178 cases. Pediatr Dermatol 1991;8(4):267-76.

6. Mulliken JB. Cutaneous Vascular Anomalies. In: Mccarthy JG, editor. Plastic Surgery: Tumors of Head and Neck and Skin. Vol. 5. Philadelphia: B Saunders Company Ltd; 1990:3194-230.

7. Neville, Brad W. Oral and Maxillofacial Pathology ( $3^{\text {rd }}$ edition). 507-19.

8. Derkenne R, Coulet O, Varoquaux A, de Biasi C, Tomasi M. Nasal cavity lobular capillary hemangioma due to insect sting. Eur Ann Otorhinolaryngol Head Neck Dis 2012;129(5):278-80.

9. Baliga MJ, Alexander S, Mallya LP, Sudha P. Capillary hemangioma- a report of a case. J Indian Soc Pedod Prev Dent 1996;14(2):36-8.

10. Rachappa MM, Triveni MN. Capillary hemangioma or pyogenic granuloma: A diagnostic dilemma. Contemp Clin Dent 2010;1(2):119-22.

11. Yoon RK, Chussid S, Sinnarajah N. Characteristics of a pediatric patient with a capillary hemangioma of the palatal mucosa: a case report. Pediatr Dent 2007;29(3):239-42.

12. Dilsiz A, Aydin T, Gursan N. Capillary hemangioma as a rare benign tumor of the oral cavity: a case report. Cases J. 2009; 2:8622

13. Lale AM, Jani P, Coleman N, Ellis PD. A palatal hemangioma in a child. J Laryngol Otol 1998;112(7):677-8.

14. Varma S, Gangavati R, Sundaresh KJ, Mallikarjuna R. Lobulated capillary hemangioma: a common lesion in an uncommon site. BMJ Case Rep 2013;2013.

15. Ozcan C, Apa DD, Görür K. Pediatric lobular capillary hemangioma of the nasal cavity. Eur Arch Otorhinolaryngol 2004;261(8):449-51.

16. Satish V, Bhat M, Maganur PC, Shah P, Biradar V. Capillary hemangioma in the maxillary anterior region: A case report. Int J Clin Pediatr Dent 2014;7(2):144-47. 